\title{
Relationship between Depressive Symptomatology, Physical Activity, and Response to Online Health Messages
}

\author{
Jason Gibbs ${ }^{1,2, *}$, Hillary E. Swann-Thomsen ${ }^{2}$ and Nicki L. Aubuchon-Endsley ${ }^{2}$ \\ 1 Rogers Behavioral Health, Nashville, TN 37205, USA \\ 2 Department of Psychology, Idaho State University, Pocatello, ID 83209, USA; \\ hillaryswannthoms@isu.edu (H.E.S.-T.); nia2175@utulsa.edu (N.L.A.-E.) \\ * Correspondence: Jason.gibbs@rogersbh.org
}

Citation: Gibbs, J.; Swann-Thomsen, H.E.; Aubuchon-Endsley, N.L. Relationship between Depressive Symptomatology, Physical Activity, and Response to Online Health Messages. Obesities 2021, 1, 144-156. https://doi.org/10.3390/

Obesities1030013

Academic Editor: Gerard A. Kennedy

Received: 30 September 2021

Accepted: 24 October 2021

Published: 27 October 2021

Publisher's Note: MDPI stays neutral with regard to jurisdictional claims in published maps and institutional affiliations.

Copyright: (c) 2021 by the authors. Licensee MDPI, Basel, Switzerland. This article is an open access article distributed under the terms and conditions of the Creative Commons Attribution (CC BY) license (https:/ / creativecommons.org/licenses/by/ $4.0 /)$.

\begin{abstract}
Although physical activity interventions are efficacious for decreasing depressive symptomatology severity, there are several barriers to accessing treatment, supporting the need for intervention delivery in more accessible and cost-effective modes. However, individuals may respond defensively to health messages if perceiving them as threatening, and thereby fail to change behaviors. Although online, health-based interventions are effective in leading to behavior change, limited research has been conducted to identify ways in which people respond differently to online health messages. Therefore, the aim of this study was to investigate how individuals differ in their acceptance and interpretation of online health-related messages as threatening, as well as their desire to improve health behaviors based on their current depressive and physical activity levels. A total of 197 participants $\left(M_{\mathrm{AGE}}=36.17\right.$ years, $S D_{\mathrm{AGE}}=12.52$ years $)$ drawn from Amazon Mechanical Turk (MTurk) were asked to read a message regarding the importance of physical activity for health. Their defensiveness and behavior change intentionality were evaluated in relation to physical activity and depressive symptomatology. Individuals who engaged in less physical activity were more accepting of the health message, more likely to find the article threatening, agree that less exercise was related to negative health issues, and agree they should increase their physical activity. Individuals with higher self-ratings of depressive symptomatology were less accepting of the health message, found the article more threatening, and believed it to be less important to increase their physical activity levels. However, neither physical activity nor depression symptom severity were related to whether an individual would actually increase their physical activity. There was no statistically significant correlation between physical activity and depression. Explanations for these findings are provided.
\end{abstract}

Keywords: physical activity; depression; obesity; online health messages

\section{Introduction}

Health message delivery is key to providing vital health information and encouraging individuals to make positive health behavior changes. However, health messages that target behavior can result in negative responses if readers experience a threat to their selfidentity [1-5]. Specifically, defensive responding to online health messages may include less acceptance of the message, greater perceived threat, and less intent to change, which are all fundamental to health behavior change [6]. Therefore, finding ways to spread health messages in a manner that is well-received is vital. This is particularly important given that these messages may be widely disseminated, particularly via online platforms [7]. However, more research is needed to understand prospective moderating and mediating factors influencing relations among health message viewing, interpretation, and important health outcomes. While contemporary literature suggests that gain-framed messages may be more persuasive and adaptive [8], understanding possible mechanisms among which loss-framed messages fail to promote positive behavior change may assist in identifying future intervention targets to increase the health and wellbeing of those receiving the 
health messages. Given the high prevalence, lasting and widespread negative impact, and interacting sequelae of several major public health concerns, including obesity and mental health symptoms, the current study focused on a common risk factor for both, physical activity [9].

\subsection{Physical Activity, Behavior, and Health Outcomes}

Rapid changes within the U.S. have resulted in more time spent indoors, which may be one of several risk factors for increasingly sedentary lifestyles, lack of engagement in physical activity, and increased obesity rates [9]. Engagement in physical activity is associated with a range of benefits, such as decreased risk of cardiovascular disease [10], depression [11], anxiety [12], and cancer [13]. However, motivating individuals to engage in physical activity is difficult [14,15]. Approximately $47 \%$ of adults meet neither the aerobic nor muscle-strengthening guidelines according to 2008 federal physical activity guidelines for Americans [16].

In addition, attrition rates for sustained and intermittent exercise programs are estimated to vary between 7-58\% [17]. Individuals often express a lack of motivation for engaging in fitness activities due to a variety of factors, such as fatigue/amotivation and obligations/lack of time [14]. Furthermore, negative information processing can lead to reduced likelihood of engaging in physical activity, especially among those who are most in need of it. For example, overweight individuals are more likely to internalize negative societal attitudes towards themselves. Specifically, when primed to think about weightrelated stereotypes, overweight women evidence significantly lower levels of dietary and health intentions than controls [18].

Despite common barriers to engaging in physical activity, improving the likelihood that people will engage in such activities is crucial to prevent increasingly more prevalent negative health outcomes. Unfortunately, these barriers often form a positive feedback loop, wherein individuals who are not engaging in physical activity suffer health-related setbacks, which lead to increased exercise avoidance and additional setbacks. Therefore, prevention efforts that are cost-effective, widely disseminated, and accessible (e.g., online health messages), may be the most effective for individuals at risk.

\subsection{Depression and Physical Activity}

Decreased physical activity is associated with more depression symptoms/severity. In a review of studies of adult patients [19], researchers found that baseline depression was associated with reduced levels of physical activity upon follow-up. Additionally, depressive symptoms tend to decrease after repeated episodes of aerobic exercise in adults (although the frequency/intensity of exercise varies among studies), with a large review of published studies finding a moderate effect size (i.e., pooled standard mean difference $=0.62$ ) for depressive symptom remission due to exercise, and equivalent symptom amelioration to psychotherapy and pharmacotherapy [20]. Unfortunately, due to the nature of depression, those who are most depressed may be least likely to be motivated to begin and engage in exercise [21], which may explain why the two risk factors interact in relation to negative health outcomes such as obesity. However, more work is needed to explore these risk factors in relation to responses to health messages.

\subsection{Online Health Information}

Greater access to internet health providers and information has resulted in several advantages over in-person healthcare, including lower cost, increased access and availability, reduced stigma, and greater dissemination ability [22]. Online health-based interventions have been shown to be effective at improving a range of health behaviors, with a mean effect size of Cohen's d $=0.44$ found for increasing physical activity in particular [23,24]. However, given that individuals experiencing more depression symptoms and/or challenges with weight management may have unique barriers to service utilization and treatment adherence, research is needed to examine differential response to online-based health 
messages. This is important, as understanding which individuals are least likely to respond to online health messages can increase providers' ability to identify those at greatest risk and possibly target their messages to reduce attrition and increase treatment compliance.

Thus, the aim of this study is to examine how the acceptance and interpretation of an online health message (i.e., on staying active) as threatening interacts with physical activity, depressive symptomatology, and willingness to improve one's health behavior. Due to the resistance to health message delivery people experience when their self-identity is threatened [1-5], and the reduced physical activity observed among individuals experiencing symptoms of depression [19], we hypothesize that individuals who engage in less physical activity and report experiencing more depression symptoms will be less accepting of the online-disseminated health message regarding staying active, will find it more personally threatening, and will be less likely to report intent to increase their physical activity. Finally, we hypothesized that more physical activity will be related to less depressive symptomatology, which would replicate the empirical literature within a non-clinical, Amazon Mechanical Turk (MTurk) sample [25].

\section{Materials and Methods}

\subsection{Participants}

Using convenience sampling via MTurk, data were collected from 197 participants $\left(M_{\mathrm{AGE}}=36.17\right.$ years, $S D_{\mathrm{AGE}}=12.52$ years $)$, whose sociodemographic characteristics can be seen in Table 1. This sample was compared to the U.S. Census data to assess for generalizability of our sample data to the U.S. population. The largest category of participants self-identified as white, married, and having completed a bachelor's or technical degree.

Table 1. Sociodemographic variables.

\begin{tabular}{|c|c|c|}
\hline Primary Variable & Current Study Sample \% & 2017 U.S. Census Data \% \\
\hline \multicolumn{3}{|l|}{ Race/Ethnicity } \\
\hline White/Caucasian & $83 \%$ & $73 \%$ \\
\hline Black or African American & $7 \%$ & $13 \%$ \\
\hline Asian American & $9 \%$ & $5 \%$ \\
\hline American Indian or Alaskan Native & $2 \%$ & $1 \%$ \\
\hline \multicolumn{3}{|l|}{ Marital Status } \\
\hline Single/Never Married & $32 \%$ & $30 \%$ \\
\hline Married & $46 \%$ & $46 \%$ \\
\hline Divorced & $6 \%$ & $12 \%$ \\
\hline Widowed & $1 \%$ & $9 \%$ \\
\hline \multicolumn{3}{|l|}{ Education } \\
\hline Grade school & $1 \%$ & $13 \%$ \\
\hline High school & $10 \%$ & $27 \%$ \\
\hline Some college & $23 \%$ & $21 \%$ \\
\hline Associate's degree & $12 \%$ & $8 \%$ \\
\hline Bachelor's or technical degree & $38 \%$ & $19 \%$ \\
\hline Graduate or professional degree & $18 \%$ & $12 \%$ \\
\hline
\end{tabular}

\subsection{Procedure}

The Idaho State University (ISU) Human Subjects Committee approved this study. Participants were recruited through MTurk, a crowdsourcing internet marketplace that enables recruitment via an online format. Individuals recruited from this medium tend not to fundamentally differ from the general U.S. population [26]. Participants were instructed to read the informed consent form (see Appendix A), then provided their MTurk Worker ID to receive nominal payment (USD 0.50) if they completed a minimum of $80 \%$ of the protocol with at least $80 \%$ correctness on attention checks. 
Then, participants were asked to read a short article (health message) on the risks of not exercising titled "Staying Active" from the Harvard School of Medicine (see Appendix B). Participants then completed the self-resources manipulation. Participants were assigned to a self-resource manipulation. However, the manipulation was unsuccessful and excluded from analyses. Following this, participants were queried about the article and asked to respond to five questions on a 9-point scale ranging from "Low" to "High," including: "To what extent do you agree or disagree with the article that low levels of exercise increase health problems and shorten the length of one's life", "How important do you think it is for people to increase their level of exercise in order to avoid the poor outcomes and reduced life span", "To what degree is the article personally threatening to you", "To what extent do you think that you, personally, should increase your amount of daily physical activity", and "To what extent do you think that you, personally, will actually increase your daily physical activity?" Participants then completed self-report measures, including the Patient Health Questionnaire [27] (PHQ-9), International Physical Activity Questionnaire-Short Form-Self-Report Version [28] (IPAQ-SF), and demographic questions (see Appendix C). Finally, a debriefing statement (see Appendix D) was provided.

\subsection{Measures}

\subsubsection{Patient Health Questionnaire-9 (PHQ-9)}

The PHQ-9 is a widely used and validated 9-item self-report measure assessing symptoms of depression that correspond to DSM-IV-TR criteria for Major Depressive Disorder [27] (MDD). The PHQ-9 was used due to its brevity, sound psychometric properties (i.e., sensitivity and specificity $=0.88$ ), and widespread utilization. Total scores were used in correlation analyses.

\subsubsection{International Physical Activity Questionnaire-Short Form-Self-Report Version (IPAQ-SF)}

The IPAQ-SF is a widely used and validated (i.e., good content validity; total score test-retest reliability from 3-7 days, $r=0.75-1.00$; construct validity with accelerometer data, $r=0.52-0.55), 7$-item self-report measure that assesses activity at three intensity levels over the past 7 days [29]. The IPAQ-SF was used due to the brevity of the measure and the inability to use behavioral measures of physical activity in an online sample. Scores can be represented via distributions into each of the three categories or as descriptive statistics for the total weekly physical activity duration in minutes (across all intensity levels), the latter of which was used in correlation analyses.

\subsection{Data Analyses}

Data were analyzed using the Statistical Package for the Social Sciences (SPSS) software (Version 27.0, IBM Corp, Armnok, NY, USA). Descriptive statistics were utilized to describe primary study variables (see Tables 2-4). Two-tailed tests using an alpha value of 0.05 were adopted to examine the statistical significance of all inferential statistics. A chi-square goodness of fit test was conducted to ascertain if the current sample sociodemographic data approximated that of the 2017 U.S. Census data (see Table 1), similar to prior reports regarding the sociodemographic makeup of MTurk workers. Pearson's product moment correlations were used to evaluate associations among study variables. 
Table 2. Five-question survey descriptive statistics.

\begin{tabular}{|c|c|c|c|c|c|}
\hline & $\begin{array}{l}\text { To What Extent Do You } \\
\text { Agree or Disagree with the } \\
\text { Article that Low Levels of } \\
\text { Exercise Increase Health } \\
\text { Problems and Shorten the } \\
\text { Length of One's Life? }\end{array}$ & $\begin{array}{l}\text { How Important Do You } \\
\text { Think It Is for People to } \\
\text { Increase Their Level of } \\
\text { Exercise in Order to Avoid } \\
\text { Poor Health Outcomes } \\
\text { and Reduced Life Span? }\end{array}$ & $\begin{array}{l}\text { To What Extent Do } \\
\text { You Think that You, } \\
\text { Personally, Should } \\
\text { Increase Your } \\
\text { Amount of Daily } \\
\text { Physical Activity? }\end{array}$ & $\begin{array}{l}\text { To What Extent Do } \\
\text { You Think that You, } \\
\text { Personally, Will } \\
\text { Actually Increase Your } \\
\text { Daily Physical } \\
\text { Activity? }\end{array}$ & $\begin{array}{c}\text { To What Extent Was } \\
\text { the Article } \\
\text { Personally } \\
\text { Threatening to You? }\end{array}$ \\
\hline$n$ & 195 & 197 & 196 & 196 & 197 \\
\hline$M$ & 7.26 & 7.97 & 6.88 & 6.10 & 3.43 \\
\hline$S D$ & 2.023 & 1.499 & 2.184 & 2.149 & 2.657 \\
\hline
\end{tabular}

Table 3. IPAQ-SF descriptive statistics.

\begin{tabular}{cc}
\hline Category & Participant Frequency $(\boldsymbol{n})$ or Duration $\boldsymbol{M}(\mathbf{S D})$ \\
\hline Low Physical Activity & 41 \\
Medium Physical Activity & 42 \\
High Physical Activity & 115 \\
All Physical Activity (Weekly Duration) & $M=17.10 \mathrm{~h}(S D=15.12 \mathrm{~h})$ \\
\hline
\end{tabular}

Table 4. PHQ-9 descriptive statistics.

\begin{tabular}{ccc}
\hline$n$ & Mean & (SD) \\
\hline 195 & 5.487 & 6.083 \\
\hline
\end{tabular}

\section{Results}

\subsection{Descriptive StatisticsT}

Mean five-question survey answers ranged from 3.43 to 7.97 on a 1 to 9 scale, with the lowest ratings regarding the degree to which the article was personally threatening and the highest rating for agreement that people should increase their exercise for better health outcomes and longevity. The average PHQ-9 score was 5.49, which fell within the mild depression range, though there was notable variability in self-reported depression symptom severity (i.e., $S D=6.08$; See Table 4 ). Most participants (58\%) obtained scores on the IPAQ-SF suggestive of high activity, though roughly $21 \%$ of participants obtained activity scores within the low and medium ranges each (see Table 3 ).

\subsection{Participants Compared to Census Data}

When comparing current study participant data to 2017 U.S. Census data, regarding race/ethnicity, the current sample was statistically significantly different from the U.S. Census data, $\chi^{2}(3)=12.34, p=0.006$. Investigation of Table 1 suggests that this may be due to a larger percentage of white participants in the current sample with percentages approximately half the size in the current sample of individuals identifying as BIPOC compared to the 2017 U.S. Census sample. Likewise, for marital status, the current sample was statistically significantly different from the 2017 U.S. Census data, $\chi^{2}(3)=20.70$, $p<0.001$. While there are similar percentages of single and married participants between samples, the percentage of divorced participants was twice as high, and the percentage of widowed participants was nine times as high in the 2017 U.S. Census sample compared to the current study sample. Lastly, in reference to education, the current sample was also statistically significantly different from the 2017 U.S. Census data, $\chi^{2}(5)=95.69, p<0.001$. Specifically, a larger percentage of current study participants (68\%) completed college degrees in comparison to the 2017 U.S. Census participants (39\%).

\subsection{Physical Activity and Response to Health Message}

Participants with lower average physical activity were more likely to agree with the article than those who endorsed higher average physical activity $(r=-0.15, p=0.035)$. Participants also were more likely to find the article more personally threatening if they 
indicated lower physical activity than those who engaged in higher average physical activity $(r=-0.17, p=0.016)$. However, there was not a significant correlation between actual physical activity and perceptions of the importance of physical activity $(r=-0.03$, $p=0.67)$. Lastly, regarding behavioral intentionality, individuals with less average physical activity endorsed that they should increase their level of physical activity $(r=-0.24$, $p=0.001$ ), but there was not a significant correlation between actual physical activity and participants endorsing that they would increase their physical activity $(r=0.06, p=0.391)$.

\subsection{Depression and Response to Health Message}

Individuals endorsing greater depression symptom severity indicated that it was less important for people to increase physical activity to avoid poor health outcomes $(r=-0.22$, $p=0.002)$ and found the article to be more personally threatening than those with lower PHQ-9 scores $(r=0.25, p<0.001)$. Additionally, individuals with greater depression symptom severity endorsed that they should increase their physical activity $(r=0.14$, $p=0.046)$. However, there was not a significant correlation between depressive symptom severity and agreement with the article's message $(r=-0.04, p=0.063)$ or that one would increase their physical activity $(r=-0.007, p=0.92)$.

\subsection{Depression and Physical Activity}

There was not a significant correlation between physical activity and depression in the study sample $(r=-0.03, p=0.68)$.

\section{Discussion}

\subsection{Participants}

Descriptive statistics suggest adequate variability in ratings across measures used, including the finding that the average PHQ-9 score for the sample was in the mild depression range. Moreover, over half of the participants reported engaging in high levels of physical activity. This information, coupled with the fact that, in comparison to U.S. Census data, more participants in the current study were college educated, less were widowed or divorced, and more identified as caucasian or white, suggests that they may be at relatively lower risk for mental and physical health inequities, supporting future studies with more diverse participants.

\subsection{Health Message Acceptance and Threat Perception}

Contrary to our hypotheses, less self-reported engagement in weekly physical activity was associated with greater acceptance of the health message regarding staying active. This may be as those who engage in less physical activity are more likely to have health concerns, and therefore this association seems more salient and results in a more robust relationship, even if they found the health message to be more threatening. It may also be that those who exercise less are more aware of their need to increase their physical activity in the future. This suggests that they are informed of physical activity guidelines and where their activity levels should and do fall.

However, the same pattern of results did not hold true for participants who reported experiencing more depressive symptoms. As originally hypothesized, higher self-reported depressive symptomatology was correlated with greater health message threat interpretation and less endorsement of the notion that physical activity is necessary to lead a healthy lifestyle. The first finding is consistent with cognitive theories of depression that suggest that those who experience depression are more likely to view themselves, the environment, and the future in a more negative way [30]. The second finding may be due to the increased levels of pessimism observed in those experiencing greater depression symptom severity [31], which could reduce their belief that physical activity would be helpful. Research has suggested that individuals who endorse higher levels of pessimism are more likely to engage in low or no physical activity [32]. This is consistent with existing research results, suggesting that individuals most at risk for problematic health outcomes are most likely to 
feel threatened and reject the delivery of the health message and, therefore, any subsequent behavior change [33].

\subsection{Behavioral Intentionality and Links between Physical and Mental Health}

Physical activity and depression symptom severity were only linked to the belief that one should increase their physical activity, but not that one will increase their physical activity, though actual physical activity relationships to behavioral intentionality evidenced the opposite predicted direction of relations. Regarding physical activity, while less engagement in physical activity was correlated with greater health message defensiveness, it was associated with greater acceptance of the message and associated intention to engage in more physical activity. This could be attributed to the fact that those individuals who are already engaging in an appropriate amount of exercise felt that they were meeting their daily fitness needs and therefore did not see a need to exercise more, whereas those who were not engaging in enough physical activity were cognizant of this fact. This suggests that this population of low exercisers might be insightful about their need to change but may encounter barriers to doing so. In terms of depression, this finding replicates others in the literature. For example, researchers [21] found similar rates of willingness to engage in exercise in a group of control individuals and those with likely depression. It may be that the defensiveness and resistance to understanding the health message does not significantly impact planned behavior, just the behavior itself.

The lack of statistically significant correlation between physical activity and depression is surprising, considering their negative relationship supported in the literature [20]. One possibility for these findings is the correlational nature of the study. While active participation in exercise may help alleviate depression, perhaps members of this sample who were already more depressed were more likely to be engaging in exercise for other reasons not evaluated by this study (e.g., individuals with work, family, or other obligations involving mandatory physical activity or athletes undergoing stressful competition). Additionally, it may be that as we had a limited range of PHQ-9 scores, this restricted our correlation coefficient and weakened a possible association that has been demonstrated in previous studies.

\subsection{Practical Implications}

Taken together, these findings have potential clinical implications, though additional research would be needed to support the clinical utility of findings based on current study limitations. First, those engaging in less physical activity were more likely to agree with the article and report intentions to improve upon their physical activity, despite their feelings of threat. This suggests that, as long as individuals do not experience elevated depression symptomatology that may impact how they perceive and interpret health messages, problem-solving other barriers to physical activity in an overt and candid manner may work to increase physical activity and assist with addressing important community health goals, such as reduction in the proportion of individuals classified as overweight or obese. For those who are experiencing elevated depressive symptoms, it may be important to frame health messages in a way that is less threatening to shift receptivity to messages about health behavior change. For example, perhaps the individual could be asked to identify a health goal and the message could be adjusted to target this goal (e.g., targeting symptoms of depression rather than weight loss). This could be facilitated by using empirically supported treatments for health behavior change such as Motivational Interviewing, which may be particularly helpful for adapting the intervention to clients fluid insight, goals, and self-efficacy regarding exercise, despite expected resistance and barriers [34].

\subsection{Study Limitations and Future Directions}

There were several limitations to the current study. First, a behavioral measure of physical fitness was not used. Participant self-reports on the IPAQ-SF are often discrepant 
from behavioral physical activity measurements [28], leading to potential error in measurement of actual involvement in physical activities. Therefore, data should be interpreted with this in mind, as it could limit generalizability. Additionally, specific groups of individuals reporting physical activity levels (low, medium, and high) were not used in current study analyses, which could reduce clarity of results (e.g., perhaps acceptance/resistance to health messages varies based on whether individuals engage in low, medium, or high levels of physical activity).

Another limitation surrounds the generalizability of findings. Indeed, although the sample of individuals participating in MTurk are similar to other online recruiting sources, important demographic differences among them are often observed [35]. These differences were replicated in our study, with our sample's marital status, education, and ethnicity more closely matching that of MTurk than other online recruiting sources or the general U.S. population (see Table 1). As noted above, our sample was more highly educated, white, and less likely to be widowed or divorced, and therefore the findings of this study may pertain more so to this specific sample than the general U.S. population.

Finally, the reflection questions asked of participants in this study (e.g., "To what extent do you agree or disagree with the article that low levels of exercise increase health problems and shorten the length of one's life") were chosen due to their face validity, as opposed to selecting questions from an established and empirically validated assessment. Therefore, no psychometric data on the appropriateness of these questions is available.

\section{Conclusions}

Taken together, there may be some unique cognitive and affective patterns in those experiencing more depression symptoms that not only negatively impact their defensiveness to health messages, but also their willingness to accept health-promoting information. While individuals who engage in less physical activity may be more defensive to messages about physical activity, they are still willing to accept messages that link physical activity and improved health. Future research should utilize behavioral observations of physical activity by trained observers or via technological monitoring (e.g., Fitbits) after the implementation of virtual health information interventions. Relatedly, it may be helpful to specifically group participants by their levels of physical activity (low, medium, and high) to examine differences in acceptance and resistance to health message information. Moreover, future research should continue to explore ongoing barriers to increasing one's physical activity even in the face of the knowledge of one's need for and intention for greater physical activity. Clarification may provide new insights into techniques that providers can use to increase exercise adherence. Future research should also investigate how to successfully convey health messages to individuals suffering from higher levels of depression. Not only do the results of this study suggest they may be more likely to interpret the health messages as threatening and may view it as less important to their well-being, but these individuals may be most in need of exercise interventions to reduce their symptom severity. Finally, future research should identify how to best implement long-term change in individuals' physical activity levels via online interventions, particularly given that sustained physical activity is more predictive of positive mental and physical health outcomes [34]. Currently, improvements in physical activity after the implementation of online-based health information procedures are often short-lived, with individuals tending to regress back toward pre-treatment levels of activity [23], suggesting that relapse prevention and maintenance could be helpful targets for future investigation. 
Author Contributions: Conceptualization, J.G., H.E.S.-T. and N.L.A.-E.; methodology, J.G., H.E.S.-T. and N.L.A.-E.; formal analysis, H.E.S.-T.; investigation, H.E.S.-T. and N.L.A.-E.; resources, N.L.A.-E.; data curation, H.E.S.-T.; writing-original draft preparation, J.G., H.E.S.-T., and N.L.A.-E.; writingreview and editing, J.G., H.E.S.-T. and N.L.A.-E.; supervision, N.L.A.-E.; and funding acquisition, N.L.A.-E. All authors have read and agreed to the published version of the manuscript.

Funding: This research was partially funded by an Institutional Development Award (IDeA) from the National Institute of General Medical Sciences of the National Institutes of Health under Grant \#P20GM103408.

Institutional Review Board Statement: The study was conducted according to the guidelines of the Declaration of Helsinki and approved by the Institutional Review Board of Idaho State University (IRB Registration \#: IRB00004102; Protocol \# IRB-FY2016-362, 11/30/2016).

Informed Consent Statement: Informed consent was obtained from all subjects involved in the study.

Data Availability Statement: Data available upon request to the corresponding author.

Acknowledgments: The authors thank Jared Vineyard and Joe Neal for their contributions to the initial study design, as well as Neal for his efforts on an initial draft of this manuscript and Vineyard for his assistance with IRB approval and data collection. We would also like to thank all research assistants and participants who contributed to this work.

Conflicts of Interest: The authors declare no conflict of interest.

\section{Appendix A}

ISU Human Subjects Committee Informed Consent Form for Non-Medical Research CONSENT TO PARTICIPATE IN RESEARCH

\section{PURPOSE OF THE STUDY}

The study is designed to understand the relationship between self-resources including self-reflection have on attitudes towards health messages.

\section{PROCEDURES}

If you volunteer to participate in this, you will be asked to complete several surveys, write about your experiences and read information about exercise. It is anticipated that this process will take approximately $1 / 2 \mathrm{~h}$ to complete. The surveys contain questions about you and your attitudes.

\section{POTENTIAL RISKS AND DISCOMFORTS}

There are minimal risks and discomforts associated with participation in this study. If you are uncomfortable about answering any of the questions, you may skip those questions or withdraw from the study without any penalty.

\section{ANTICIPATED BENEFITS TO SUBJECTS}

Participants will receive nominal payment for their participation, and increased knowledge of the psychological research process.

\section{ANTICIPATED BENEFITS TO SOCIETY}

The study may potentially lead to a better understanding of how personal psychological resources are related to health message processing.

\section{ALTERNATIVES TO PARTICIPATION}

Not applicable.

\section{PAYMENT FOR PARTICIPATION}

Individuals participating will receive a nominal payment (\$0.50) for completing a minimum of $80 \%$ of the Human Intelligence Task with a minimum of an $80 \%$ pass rate on attention checks. 


\section{FINANCIAL OBLIGATIONS}

Not applicable.

\section{PRIVACY AND CONFIDENTIALITY}

No personal information about you, including your name and any identifying information, will be recorded on the survey and questionnaires. You will be given a study identification number and this number will not be linked to any personal information. In other words, no data are linked to any personal information. All data collected for this study will be kept confidential. They will be collected and stored electronically on password protected hard drives owned and/or maintained by the principal investigator of this research. Only the principal investigator and trained research assistants will have access to data. Data without any way to identify who supplied the data may be posted on websites like the Open Science Framework.

\section{PARTICIPATION AND WITHDRAWAL}

Your participation in this research is COMPLETELY VOLUNTARY. If you choose not to participate, that will not affect your relationship with ISU, or your right to receive services at ISU to which you are otherwise entitled. If you decide to participate, you are free to withdraw your consent and discontinue participation at any time without prejudice to your future at ISU.

\section{CONSEQUENCES OF WITHDRAWAL}

If you decide to withdraw from the study, you will not be penalized. You will only receive the nominal payment if you complete at least $80 \%$ of the procedure with adequate performance on attention check questions.

\section{IDENTIFICATION OF INVESTIGATORS}

In the event of a research related injury or if you experience an adverse reaction, please immediately contact the principal investigator, ISU, at ISU. If you have any questions about the research, please feel free to contact Dr. Aubuchon-Endsley.

\section{RIGHTS OF RESEARCH SUBJECTS}

You may withdraw your consent at any time and discontinue participation without penalty. You are not waiving any legal claims, rights, or remedies because of your participation in this research study. If you have any questions regarding your rights as a research subject, you may contact the ISU Human Subjects Committee office at 208-282-2179 or by writing to the Human Subjects Committee at Idaho State University, Mail Stop 8046, Pocatello, ID 83209.

\section{CONSENT OF RESEARCH SUBJECT}

Please select the corresponding box. I have read the information provided above and I willingly agree to participate in the research it describes.

\section{YES NO}

\section{Appendix B}

Health message on the risks of not exercising from the Harvard School of Medicine Staying Active

In addition to eating high-quality foods, physical activity can help you reach and maintain a healthy weight.

- Getting regular physical activity is one of the best things you can do for your health It lowers the risk of heart disease, diabetes, stroke, high blood pressure, osteoporosis, and certain cancers, and it can also help control stress, improve sleep, boost mood, keep weight in check, and reduce the risk of falling and improve cognitive function in older adults. 
- It doesn't take marathon training to see real health gains. A 30-min brisk walk on five days of the week is all most people need. Getting any amount of exercise is better than none.

- Being a "couch potato" may be harmful even for people who get regular exercise. Regular physical activity helps the body function better-it keeps heart disease, diabetes, and a host of other diseases at bay, and is a key component for losing weight.

- The precise amount of exercise needed to achieve or maintain a healthy weight varies based on a person's diet and genes. The American College of Sports Medicine and the American Heart Association support the idea that "more activity increases the probability of success".

- How much exercise do you need? Physical activity guidelines-including strength training and flexibility training - explain how much you should be moving. Keep exercise safety in mind, too.

Physical activity can also help people maintain weight loss.

- Among the nearly 3700 men and women who are part of the National Weight Control Registry, a group that includes only people who lost more than 30 pounds and kept them off for at least a year, the average participant burns an average of about 400 calories per day in physical activity. That's the equivalent of about 60 to $75 \mathrm{~min}$ of brisk walking each day, or 35 to 40 min of daily jogging. But there's quite a bit of variation from participant to participant-some require more physical activity to keep the weight off, some require less. The Cost of Inactivity Exercise and physical activity benefit the body, while a sedentary lifestyle does the opposite-increasing the chances of becoming overweight and developing a number of chronic diseases.

- Only about 30 percent of adult Americans report they get regular physical activity during their leisure time-and about 40 percent of Americans say they get no leisuretime physical activity at all.

- The Nurses' Health Study found a strong link between television watching and obesity. Researchers followed more than 50,000 middle-aged women for six years, surveying their diet and activity habits. Findings showed that for every two hours the women spent watching television each day, they had a 23 percent higher risk of becoming obese and 14 percent higher risk of developing diabetes. It didn't matter if the women were avid exercisers: The more television they watched, the more likely they were to gain weight or develop diabetes, regardless of how much leisure-time activity and walking they did. Long hours of sitting at work also increased the risk of obesity and diabetes.

- $\quad$ Researchers at Tokyo Medical University found an association between spending less time watching television and a lower risk of overweight and obesity in older adults, regardless of whether participants met physical activity guidelines. The study followed 1806 participants between the ages of 65 and 74. Participants were put into one of four categories based on television viewing time. The less time spent watching television, the lower the participants' risk of becoming overweight or obese.

- Another study analyzed the global effect of inactivity on the increase of diseases. The researchers estimated that physical inactivity accounts for $6 \%$ of the burden of heart disease, $7 \%$ of type 2 diabetes, $10 \%$ of breast cancer, and $10 \%$ of colon cancer. Inactivity also causes $9 \%$ of premature mortality. These staggering statistics put the true dangers associated with inactivity into a global perspective.

\section{Sit Time}

- More recently, studies have found that people who spend more time each day watching television, sitting, or riding in cars have a greater chance of dying early than people who are more active. Researchers speculate that sitting for many hours may change peoples' metabolism in ways that promote obesity, heart disease, diabetes, and other chronic conditions. It is also possible that sitting is a marker for a broader sedentary lifestyle. Furthermore, staying active does not mitigate the harmful effects of sit time. 
As you plan your daily activity routine, remember that cutting down on "sit time" may be just as important as increasing "fit time".

\section{Appendix C}

Demographics Questionnaire

What is your age?

What is gender?

What is the highest level of education you have completed?

What is your marital status?

What is your religious preference?

What ethnicity(s) do you identify as?

What is your age?

\section{Appendix D}

Debriefing Statement

Thank you very much for participating in this study. We sincerely appreciate your time and effort. The current study aims to understand how evoking personal resources can reduce defensiveness and increase acceptance of health messages. We are specifically interested in comparing different processes in evoking these personal resources such as self-affirmation and nostalgia. We believe a better understanding of these different methods can be used to improve the delivery of important health messages to the public at large. Further we wanted to understand how previously established effects in the literature could be applied to physical activity.

If you have any questions about the study, please feel free to contact the principal investigator, Jared Vineyard, Ph.D. (jvineyard@gmail.com) at Idaho State University.

\section{References}

1. Aronson, E. Dissonance theory: Progress and problems. In Theories of Cognitive Consistency: A Sourcebook; Abelson, R.P., Aronson, E., McGuire, W.J., Newcomb, T.M., Rosenberg, M.J., Tannenbaum, P.H., Eds.; Rand McNally: Chicago, IL, USA, 1968; pp. 5-27.

2. Brown, S.; Locker, E. Defensive responses to an emotive anti-alcohol message. Psychol. Health 2009, 24, 517-528. [CrossRef]

3. Kunda, Z. The case for motivated reasoning. Psychol. Bull. 1990, 108, 480-498. [CrossRef] [PubMed]

4. Steele, C.M. The psychology of self-affirmation: Sustaining the integrity of the self. In Advances in Experimental Social Psychology; Berkowitz, L., Ed.; Academic Press: New York, NY, USA, 1988; Volume 21, pp. 261-302.

5. Sweeney, A.M.; Moyer, A. Self-affirmation and responses to health messages: A meta-analysis on intentions and behavior. Health Psychol. 2015, 34, 149-159. [CrossRef] [PubMed]

6. Epton, T.; Harris, P.R.; Kane, R.; van Koningsbruggen, G.M.; Sheeran, P. The impact of self-affirmation on health-behavior change: A meta-analysis. Health Psychol. 2015, 34, 187. [CrossRef] [PubMed]

7. Berland, G.K.; Elliott, M.N.; Morales, L.S.; Algazy, J.I.; Kravitz, R.L.; Broder, M.S.; Watkins, K.E. Health information on the Internet: Accessibility, quality, and readability in English and Spanish. J. Am. Med. Assoc. 2001, 285, 2612-2621. [CrossRef] [PubMed]

8. Ort, A.; Fahr, A. Using efficacy cues in persuasive health communication is more effective than employing threats-An experimental study of a vaccination intervention against Ebola. Br. J. Health Psychol. 2018, 23, 665-684. [CrossRef] [PubMed]

9. Lincoln, K.D. Social stress, obesity, and depression among women: Clarifying the role of physical activity. Ethn. Health 2019, 24, 662-678. [CrossRef] [PubMed]

10. Lavie, C.J.; Ozemek, C.; Carbone, S.; Katzmarzyk, P.T.; Blair, S.N. Sedentary behavior, exercise, and cardiovascular health. Circ. Res. 2019, 124, 799-815. [CrossRef] [PubMed]

11. Stanton, R.; Reaburn, P. Exercise and the treatment of depression: A review of the exercise program variables. J. Sci. Med. Sport 2014, 17, 177-182. [CrossRef] [PubMed]

12. Asmundson, G.J.; Fetzner, M.G.; DeBoer, L.B.; Power, M.B.; Otto, M.W.; Smits, J.A. Let's get physical: A contemporary review of the anxiolytic effects of exercise for anxiety and its disorders. Depress. Anxiety 2013, 30, 362-373. [CrossRef] [PubMed]

13. Kruk, J.; Czerniak, U. Physical activity and its relation to cancer risk: Updating the evidence. Asian Pac. J. Cancer Prev. 2013, 14, 3993-4003. [CrossRef] [PubMed]

14. Portela-Pino, I.; Lopez-Castedo, A.; Martinez-Patiño, M.J.; Valverde-Esteve, T.; Dominguez-Alonso, J. Gender differences in motivation and barriers for the practice of physical exercise in adolescence. Int. J. Environ. Res. Public Health 2020, 17, 168. [CrossRef] [PubMed]

15. Wulf, G.; Freitas, H.E.; Tandy, R.D. Choosing to exercise more: Small choices increase exercise engagement. Psychol. Sport Exerc. 2014, 15, 268-271. [CrossRef] 
16. Blackwell, D.L.; Lucas, J.W.; Clarke, T.C. Summary health statistics for US adults: National health interview survey, 2012. Vital Health Stat. 10 2014, 260, 1-161.

17. Linke, S.E.; Gallo, L.C.; Norman, G.J. Attrition and adherence rates of sustained vs. Intermittent exercise interventions. Ann. Behav. Med. 2012, 42, 197-209. [CrossRef]

18. Seacat, J.D.; Mickelson, K.D. Stereotype threat and the exercise/dietary health intentions of overweight women. J. Health Psychol. 2009, 14, 556-567. [CrossRef]

19. Roshanaei-Moghaddam, B.; Katon, W.J.; Russo, J. The longitudinal effects of depression on physical activity. Gen. Hosp. Psychiatry 2009, 31, 306-315. [CrossRef]

20. Cooney, G.M.; Dwan, K.; Greig, C.A.; Lawlor, D.A.; Rimer, J.; Waugh, F.R.; Mead, G.E. Exercise for depression. Cochrane Database Syst. Rev. 2013, 9, 1-129.

21. Busch, A.M.; Ciccolo, J.T.; Puspitasari, A.J.; Nosrat, S.; Whitworth, J.W.; Stults-Kolehmainen, M. Preferences for exercise as a treatment for depression. Ment. Health Phys. Act. 2016, 10, 68-72. [CrossRef]

22. Griffiths, F.; Lidenmeyer, A.; Powell, J.; Lowe, P.; Thorogood, M. Why are health care interventions delivered over the internet? A systematic review of the published literature. J. Med. Internet Res. 2006, 8, e10. [CrossRef]

23. Vandelanotte, C.; Spathonis, K.M.; Eakin, E.G.; Owen, N. Website-delivered physical activity interventions: A review of the literature. Am. J. Prev. Med. 2007, 33, 54-64. [CrossRef]

24. Spittaels, H.; De Bourdeaudjuij, I.; Vandelanotte, C. Evaluation of a website-delivered computer-tailored intervention for increasing physical activity in the general population. Prev. Med. 2007, 44, 209-217. [CrossRef]

25. Ströhle, A. Physical activity, exercise, depression and anxiety disorders. J. Neural Transm. 2009, 116, 777-784. [CrossRef]

26. Levay, K.E.; Freese, J.; Druckman, J.N. The demographic and political composition of Mechanical Turk. SAGE Open 2016, 6, 1-17. [CrossRef]

27. Kroenke, K.; Spitzer, R.L.; Williams, J.B.W. The PHQ-9: Validity of a brief depression severity measure. J. Gen. Intern. Med. 2001, 16, 606-613. [CrossRef] [PubMed]

28. Hagstromer, M.; Oja, P.; Sjostrom, M. The International Physical Activity Questionnaire (IPAQ): A study of concurrent and construct validity. Public Health Nutr. 2006, 9, 755-762. [CrossRef] [PubMed]

29. van Poppel, M.N.M.; Chinapaw, M.J.M.; Mokkink, L.B.; van Mechelen, W.; Terwee, C.B. Physical activity questionnaires for adults: A systematic review of measurement properties. Sports Med. 2010, 40, 565-600. [CrossRef]

30. Gotlib, I.H.; Joormann, J. Cognition and depression: Current status and future directions. Annu. Rev. Clin. Psychol. 2010, 27, 285-312. [CrossRef] [PubMed]

31. Dickson, J.M.; Moberly, N.J.; O’Dea, C.; Field, M. Goal fluency, pessimism, and disengagement in depression. PLoS ONE 2016, 11, e0166259. [CrossRef]

32. Kavussanu, M.; McAuley, E. Exercise and Optimism: Are highly active individuals more optimistic? J. Sport Exerc. Psychol. 1995, 17, 246-258. [CrossRef]

33. Sherman, D.A.K.; Nelson, L.D.; Steele, C.M. Do messages about health risks threaten the self? Increasing the acceptance of threatening health messages via self- affirmation. Personal. Soc. Psychol. Bull. 2000, 26, 1046-1058. [CrossRef]

34. Blumenthal, J.A.; Smith, P.J.; Hoffman, B.M. Is exercise a viable treatment for depression? ACSM's Health Fit. J. 2012, 16, 14-21. [CrossRef] [PubMed]

35. Heen, M.S.J.; Lieberman, J.D.; Miethe, T.D. A comparison of different online sampling approaches for generating national samples. Cent. Crime Justice Policy 2014, 1, 1-8. 